\title{
Note on Transliteration and Orthography
}

In transcribing Indic languages, I have used the standard Devanagari transliteration system for quotations from written Hindi sources only. There is no standard orthography for Mauritian Bhojpuri, which is rarely written, and my transcriptions contain a great deal of ideolectal, generational, and register variation. I have used a modified variant of the Devanagari transliteration system for Mauritian Bhojpuri, without diacritics except for the retroflex plosives $t, t h, d$, $d h$ and the retroflex flaps $r$ and $r h$, which frequently but not consistently occur in the speech of many older and middle-aged speakers. I have also rendered the voiceless unaspirated palatal plosive represented as $c$ in standard Devanagari transliteration as $c h$ to minimize confusion for nonspecialist readers.

My transcriptions of Mauritian Creole also show considerable ideolectal and register variations combined with frequent bivalent usage, especially among Mauritian Creole-Mauritian Bhojpuri bilinguals. I have taken the orthography proposed by Baker and Hookoomsing (1987) as an orientation wherever feasible but have not adopted the use of diacritics to indicate nasalization. 
